\title{
Lifestyle modification strategies for controlling hypertension: How are these strategies recommended by physicians in Iran?
}

\author{
Sina Ahmadi ${ }^{1,2}$, Homeira Sajjadi ${ }^{1,3}$, Farhad Nosrati Nejad ${ }^{1}$, Naser Ahmadi ${ }^{4}$, Salah Eddin Karimi ${ }^{5}$, Moein Yoosefi $^{4}$, \\ Hassan Rafiey*1,2
}

Received: 4 Sep 2018

Published: 20 May 2019

\section{Abstract}

Background: This study aimed to evaluate lifestyle changes and their impact on hypertension control and why and how lifestyle modifications are recommended for patients with hypertension. Four non pharmacological strategies have been recommended by physicians for hypertension control for hypertensive patients in Iran: healthier diet, smoking cessation, physical activity, and weight loss.

Methods: Among participants of Iran STEPS Non-communicable Disease Risk Factors Survey (STEPs), 7879 hypertensive patients aged $\geq 25$ years were selected. All statistical analyses were calculated using STATA software version 14. Means, proportions, and multiple binary logistic regression models were used. Two-tailed $p$ values of $<0.05$ were considered statistically significant.

Results: The results of this study showed that about three quarters of people with high blood pressure had been recommended lifestyle strategies by their physician for blood pressure control. Of the participants, $41 \%$ reported that they had been recommended only lifestyle modification for their blood pressure (BP), while 35\% reported that they were recommended both lifestyle changes and medications as part of their treatment. Healthier diet, 71.9\% (70.8-72.9), and smoking cessation, 23.8\% (21.4-25.6), were the most and least frequently recommended strategies, respectively. Also, the rates of recommendations on smoking cessation, weight loss, increased physical activity, and healthier diet were more among women, married, and illiterate individuals, respectively.

Conclusion: Because of the changes and transitions in the lifestyle and dietary habits in the modern world, policies and training programs should be developed to improve the recommendations on lifestyle modification. Also, educational programs should be developed to increase patient's acceptance of lifestyle modifications and physical activity.

Keywords: Hypertension, Lifestyle, Physical activity, Recommendation, Non pharmacology

Conflicts of Interest: None declared

Funding: None

\section{*This work has been published under CC BY-NC-SA 1.0 license.}

Copyright $\odot$ Iran University of Medical Sciences

Cite this article as: Ahmadi S, Sajjadi H, Nosrati Nejad F, Ahmadi N, Karimi SE, Yoosefi M, Rafiey H. Lifestyle modification strategies for controlling hypertension: How are these strategies recommended by physicians in Iran? Med J Islam Repub Iran. 2019 (20 May);33:43. https://doi.org/10.47176/mjiri.33.43

\section{Introduction}

Hypertension, as a chronic disease, is a main factor in outpatient visit of physicians (1), and it is the most important risk factor for controlling coronary artery heart

\footnotetext{
Corresponding author: Dr Hassan Rafiey, ha.rafiey@uswr.ac.ir

1. Department of Social Welfare Management, University of Social Welfare and Rehabilitation Sciences, Tehran, Iran

2. Social Welfare Management Research Center, University of Social Welfare and Rehabilitation Sciences, Tehran, Iran

3. Social Determinants of Health Research Center, University of Social Welfare and Rehabilitation Sciences, Tehran, Iran

4. Non-communicable Diseases Research Center, Endocrinology and Metabolism Population Sciences Institute, Tehran University of Medical Sciences, Tehran, Iran

5. Social Determinants of Health Research Center, Health Management and Safety Promotion Research Institute, Tabriz University of Medical Sciences, Tabriz, Iran
}

disease. Also, hypertension can damage the kidneys and increase the risk of blindness and dementia (2) and is responsible for $70 \%$ of the burden of vascular heart diseases

\section{$\uparrow$ What is "already known" in this topic:}

Hypertension is one of the most common chronic cardiovascular diseases affecting more than $20 \%$ of the world's adult population. Non pharmacological strategies have been shown to help lower blood pressure and are recommended for all patients with hypertension.

$\rightarrow$ What this article adds:

About three quarters of people with high blood pressure in Iran had been recommended lifestyle strategies for blood pressure control. Physician's role is important in orienting hypertensive patients with lifestyle strategies. 


\section{(3).}

Hypertension disease, as a silent killer, is the third most common cause of death in the world due to heart coronary disease (4) and the second commonest cause of death in Iran (3). Reducing blood pressure is important because it is the most important preventable factor that can significantly reduce mortality and related illnesses. A 3milligram decrease in systolic blood pressure can reduce $8 \%$ of stroke-related deaths and $5 \%$ of deaths due to coronary artery disease. Despite effective and available antihypertensive drugs, studies showed that less than half of the patients with hypertension were receiving antihypertensive treatment and only 1 in 3 (less than $20 \%$ ) individuals had achieved blood pressure control $<140 / 90 \mathrm{mmHg}$ (2). To prevent and control hypertension, in addition to conventional therapies, nonmedical strategies, including lifestyle modifications, are also emphasized (5). Lifestyle strategies, previously labelled as nonpharmacologic therapy has significant roles in hypertensive diseases (3), and these strategies are recommended as the first step in managing blood pressure (6). One type of lifestyle interventions includes changing eating habits, such as reducing salt intake, consuming more fruits and vegetables (7), reducing fat intake, increasing potassium intake and physical activity (8), weight control (8), and smoking cessation (9).

Although the abundance of modern equipment has made life easier, they are the leading causes for physical inactivity (10) and growing prevalence of blood pressure. Thus, lifestyle interventions and changes can improve hypertensive patients' health, increase their longevity, improve their quality of life, and reduce the cost of treatment (11).

Studies show that the prevalence of hypertension in Iran is $26.6 \%$ and affects about 10 million adults, while only $25 \%$ of them receive treatment (12). Therefore, considering the importance of lifestyle in controlling and preventing hypertension, the present study aimed to investigate lifestyle strategies recommended by physicians for treatment and hypertension control in hypertensive individuals $\geq 25$-years-old.

\section{Methods \\ Study population}

This study was part of the surveillance of risk factors of non-communicable diseases in 2016 called Iranian STEPs Survey 2016, for which participants from 30 provinces of Iran were recruited. Systematic proportional sampling was used and participants were randomly recruited. Iranian men and women 25 years and older who had hypertension were included in the study for lifestyle strategy measurements. Detailed description about sampling methods is published elsewhere (13). The sample used in this study consisted of 7752 adults 25 years and older who had hypertension.

\section{Data sources}

The main data source of this study was Iran-STEPs study STEPwise, which is a cross sectional survey based on the WHO-STEPwise approach. It has been conducted on demographic, anthropometric, and biologic samples of
30541 persons aged $>18$ years in Iran.

In STEPs survey, to reduce investigator bias, all researchers were provided with training on how to fill up the questionnaire and how to store blood sample. The coordinating center supervised data gathering process to ensure the reliability and accuracy of the recorded data. All data were documented in an application that transferred data to a server as database and then a web-based dashboard was used to report and observe the purposes. Data on age, years of education, sex, place of residence, marital status, physical activity, diet, fasting plasma glucose (FPG), systolic and diastolic BP, smoking status, height, and weight were collected.

\section{Variables definition}

Hypertension was defined as systolic blood pressure equal or more than $140 \mathrm{mmHg}$ or diastolic blood pressure equal or more than 90 or use of antihypertensive medications. Lifestyle strategy was defined as presence of at least one of the recommended nonpharmacological strategies: controlling weight (reduction of excessive body weight), healthier diet, physical activity, and smoking cessation. Recommendations for healthier diet were reducing salt intake, reducing oil or fat in diet, and consuming more fruit and vegetables daily. Physical activity was defined as any combination of physical activities that exceeds 600 METs per week, and it was measured with the STEPs protocol (13). The person was considered as having normal weight if her/his body mass index (BMI) was between 18 and 25 , underweight if it was equal or less than 18 , overweight if it was between 25 and 30, and obese if it was more than 30. Based on American Diabetes Association recommendations, diabetes was considered as FPG $\geq 126 \mathrm{mg} / \mathrm{dL}$ ( $7 \mathrm{mmol} / \mathrm{L}$ ) or antihyperglycemic medication use (14). For regions variable, the country was divided into 4 large regions based on the combination of geography and socioeconomic status (15). Wealth index was constructed using principal component analysis (PCA) on household assets and dwelling characteristics, resulting in 5 quintiles from the poorest (first quintile) to the wealthiest (fifth quintile).

\section{Statistical analysis}

Prevalence of each lifestyle strategy was measured after considering sampling weights based on population size and age and gender distribution. Means and proportions with $95 \%$ confidence intervals $(\mathrm{CI})$ were calculated. Then, continuous and categorical variables were compared between different groups. Multiple binary logistic regression models were used to identify the determinants of lifestyle strategies recommended by physicians. Also, an analysis was conducted for adjusted odds ratios (OR) with $95 \%$ confidence interval to determine the associations between demographic and lifestyle strategies recommended by physicians and for each model. Nonpharmacology was calculated by proportion of those who gave a positive answer to questions related to use of lifestyle strategy. Conventional treatment was calculated in those who reported to take medications for hypertension treatment. All statistical analyses were performed using Stata software version 
14. Two-tailed $\mathrm{p}$ values of $<0.05$ were considered statistically significant.

\section{Results}

The average age of hypertensive patients in the study was $57.3 \pm 9.7$ years and $4256(54.9 \%)$ were females. Patients who said their physicians recommended healthier diet, physical activity, smoking cessation, weight loss, and nonpharmacological treatments (total) for hypertension control accounted for 3139 (40.5\%), 5574 (71.9\%), 4062 (52.4\%), 1845 (23.8\%), 2837 (36.6\%), and $5822(75.1 \%)$ of the study population, respectively. The main and most significant lifestyle modification recommended to participants with hypertension was healthier diet (71.9\%) (Table 1).

The results showed that $40.6 \%$ of the respondents reported that they used lifestyle measures exclusively to control their hypertension, while $35.4 \%$ combined nonpharmacological treatments with antihypertensive medications. Also, $18.3 \%$ of hypertensive individuals reported that they have not used any treatments to control their hypertension (Table 2).

The results indicated that recommendations were significantly associated with age and that older people had higher rates of physician recommendations to change their lifestyle, including weight control, physical activity, healthier diet, and smoking cessation, compared to younger people. According to age categories, recommendations for weight control varied from $26.9 \%$ in patients aged 25 34 to $38.9 \%$ in patients aged $45-54$ years. Also, recommendations for physical activity was more common in patients aged 55-64 years, and recommendations for healthier diet varied from $47.9 \%$ in young patients aged 25-34 years to $74.4 \%$ in oldest patients aged 65 years and older. Smoking cessation was more common in patients aged 45-54 years. The results of the present study demonstrated that the recommendations for weight control (40.6\% vs $31.7 \%$ ), physical activity (53.9\% vs $50.9 \%$ ), and healthier diet $(76.3 \%$ vs $66.6 \%)$ in women were more than in men, but recommendations on smoking cessation was less among women (11.7\% vs $31.5 \%)$. The data indicated that recommendations for weight control $(38 \%$ vs $24.1 \%$ ), physical activity ( $54.2 \%$ vs $36.7 \%$ ), healthier diet $(72.8 \%$ vs $45.4 \%)$, and smoking cessation $(26.8 \%$ vs $23.4 \%$ ) were more in married patients than in the single (Table 3).

Also, results revealed that recommendations for weight control $(38.2 \%$ vs $35.9 \%)$, physical activity (52.8\% vs $52.2 \%)$, healthier diet $(78.1 \%$ vs $69.2 \%)$, and smoking cessation $(27.1 \%$ vs $25.1 \%)$ were observed more in hyper-

Table 1. Percentage use of specific types of control strategies among adult hypertensive population $(\mathrm{n}=7.752)$

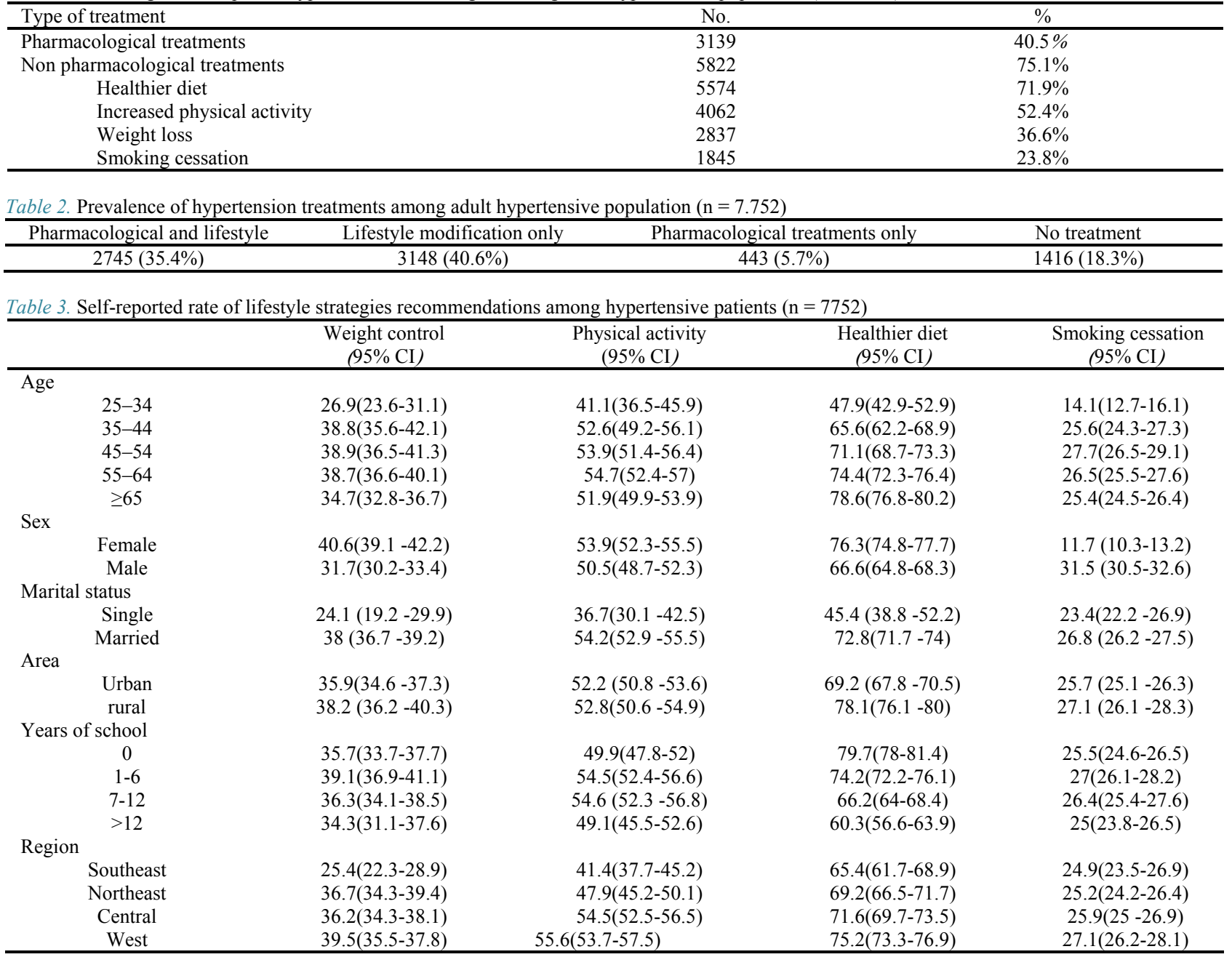


tensive patients in rural areas than in patients who lived in urban areas. Further analysis demonstrated that the most frequently reported strategy according to years of school was healthier diet (79.7\%) among illiterate individuals with hypertensive blood pressure and the least frequent strategy was smoking cessation $(25 \%)$ in hypertensive people with $>12$ years of education. Among hypertensive patients who reported a physician recommendation, all 4 recommended strategies were less among people who lived in the southeast region of the country (Table 3 ).

The results of this study showed that the chance of physician's recommendation for physical activity was $46 \%$ higher for those aged 35-44 years than for those aged 2534 years $(\mathrm{OR}=1.46 \% 95 \mathrm{CI}: 1.08-1.96)$. Also, the odds ratio for recommending a healthier diet among those aged 55-64 years was 2.38 folds $(\mathrm{OR}=2.38, \% 95 \mathrm{CI}$ : $1.72-$ $3.29)$ and it was 2.61 folds $(\mathrm{OR}=2.6, \% 95 \mathrm{CI}: 1.36-5.01)$ for smoking cessation between those aged 45-54 years, which is higher compared to those aged 25-34 years.

The results indicated that the odds ratio of physician's recommendation was $37 \%$ more for weight loss (OR = 1.37, \%95 CI: 1.61-1.56), 19\% more for physical activity $(\mathrm{OR}=1.19, \% 95 \mathrm{CI}: 1.06-1.36)$, and $62 \%$ more for healthy diet in women than in men $(\mathrm{OR}=1.62, \% 95 \mathrm{CI}$ : $1.41-$ 1.88). Also, men were more likely to be recommended to stop smoking than women $(\mathrm{OR}=0.12, \% 95 \mathrm{CI}$ : 0.08 0.16). Compared to residents of urban areas, the rural residents were $31 \%(\mathrm{OR}=1.31, \% 95 \mathrm{CI}$ : $1.15-1.50)$ more likely to be recommended by a physician to lose weight for hypertension control, $38 \%(\mathrm{OR}=1.38, \% 95 \mathrm{CI}: 1.20$ 1.60) were recommended physical activity, $78 \%(\mathrm{OR}=$ $1.87, \% 95 \mathrm{CI}: 1.22-1.58)$ healthy diet, and $36 \%(\mathrm{OR}=$ 1.36, \%95 CI: 1.01-1.85) smoking cessation. The odds ratio of a physician's recommendation to married people compared to the single people was $76.6 \%$ for weight loss $(\mathrm{OR}=1.76, \% 95 \mathrm{CI}: 1.15-2.68), 85 \%$ for physical activity $(\mathrm{OR}=1.85, \% 95 \mathrm{CI}: 1.24-2.74)$, and 2.18 folds more for healthy diet $(\mathrm{OR}=2.18, \% 95 \mathrm{CI}$ : 1.46-3.26).

The results indicated that with the increase in the education level of hypertensive patients in general, the odds of a physician's recommendation to change lifestyle became greater. Accordingly, the odds ratio of weight loss recommendation was $39 \%(\mathrm{OR}=1.39, \% 95 \mathrm{CI}: 1.12-1.72)$ in people with 12 years of education and physical activity was $36 \%(\mathrm{OR}=1.36, \% 95 \mathrm{CI}: 1.09-1.69)$ more than illiterates, while the odds of recommending a healthier diet

Table 4. Sociodemographic factors associated with physician recommended lifestyle strategies among hypertensive patients ( $\mathrm{n}=5629)$

\begin{tabular}{|c|c|c|c|c|}
\hline & Body weight control & Physical activity & Healthier diet & Smoking cessation \\
\hline $\mathrm{N}$ & OR $(95 \% \mathrm{CI})$ & OR $(95 \% \mathrm{CI})$ & OR $(95 \% \mathrm{CI})$ & OR $(95 \% \mathrm{CI})$ \\
\hline \multicolumn{5}{|l|}{ Age } \\
\hline $25-34$ & ref & ref & ref & ref \\
\hline $35-44$ & $1.23(0.91-1.68)$ & $1.46(1.08-1.96)$ & $1.51(1.11-2.07)$ & $1.79(0.89-3.58)$ \\
\hline $45-54$ & $1.07(0.79-1.43)$ & $1.33(1.01-1.77)$ & $1.62(1.20-2.20)$ & $2.61(1.36-5.01)$ \\
\hline $55-64$ & $1.07(0.79-1.44)$ & $1.43(1.07-1.92)$ & $1.73(1.27-2.36)$ & $1.96(1.02-3.77)$ \\
\hline$\geq 65$ & $1.06(0.78-1.44)$ & $1.43(1.07-1.93)$ & $2.38(1.72-3.29)$ & $1.31(0.68-2.55)$ \\
\hline \multicolumn{5}{|l|}{ Sex } \\
\hline Male & ref & ref & ref & ref \\
\hline Female & $1.37(1.21-1.56)$ & $1.19(1.06-1.36)$ & $1.62(1.41-1.88)$ & $0.12(0.08-0.16)$ \\
\hline \multicolumn{5}{|l|}{ Residence } \\
\hline Urban & ref & ref & ref & ref \\
\hline Rural & $1.31(1.15-1.50)$ & $1.38(1.20-1.60)$ & $1.87(1.58-2.22)$ & $1.36(1.01-1.85)$ \\
\hline \multicolumn{5}{|l|}{ Marital status) } \\
\hline Single & ref & ref & ref & ref \\
\hline Married & $1.76(1.15-2.68)$ & $1.85(1.24-2.74)$ & $2.18(1.46-3.26)$ & $0.98(0.41-2.35)$ \\
\hline \multicolumn{5}{|c|}{ Years of school } \\
\hline 0 & ref & ref & ref & ref \\
\hline $1-6$ & $1.15(0.98-1.35)$ & $1.19(1.03-1.38)$ & $0.93(0.78-1.13)$ & $0.89(0.65-1.23)$ \\
\hline $7-12$ & $1.17(0.96-1.41)$ & $1.27(1.05-1.53)$ & $0.78(0.63-0.97)$ & $0.67(0.45-0.99)$ \\
\hline$>12$ & $1.39(1.12-1.72)$ & $1.36(1.09-1.69)$ & $0.85(1.32-2.10)$ & $0.51(0.31-0.86)$ \\
\hline \multicolumn{5}{|l|}{ Region } \\
\hline Southeast & ref & ref & ref & ref \\
\hline Northeast & $1.59(1.26-2.01)$ & $1.17(0.94-1.46)$ & $1.26(1.01-1.62)$ & $1.27(0.76-2.1)$ \\
\hline Central & $1.57(1.24-1.97)$ & $1.53(1.23-1.91)$ & $1.50(1.17-1.92)$ & $1.61(0.96-2.73)$ \\
\hline West & $1.67(1.34-2.09)$ & $1.60(1.30-1.97)$ & $1.66(1.32-2.10)$ & $1.67(1.04-2.68)$ \\
\hline \multicolumn{5}{|l|}{ BMI } \\
\hline Normal & ref & ref & ref & ref \\
\hline Underweight & $0.51(0.28-0.92)$ & $0.68(0.44-1.06)$ & $0.75(0.46-1.21)$ & $1.24(0.59-2.65)$ \\
\hline Overweight & $1.36(1.16-1.60)$ & $1.04(0.89-1.20)$ & $1.07(0.90-1.27)$ & $0.74(0.56-0.99)$ \\
\hline Obese & $2.26(1.92-2.67)$ & $1.30(1.12-1.54)$ & $1.24(1.03-1.49)$ & $0.55(0.39-0.76)$ \\
\hline \multicolumn{5}{|l|}{ Diabetic } \\
\hline NO & ref & ref & ref & ref \\
\hline YES & $1.55(1.34-1.80)$ & $1.56(1.34-1.81)$ & $1.75(1.46-2.10)$ & $0.94(0.68-1.30)$ \\
\hline \multicolumn{5}{|l|}{ Wealth index } \\
\hline Poorest & ref & ref & ref & ref \\
\hline Richest & $1.57(1.24-2.01)$ & $1.47(1.16-1.86)$ & $1.16(0.89-1.52)$ & $0.83(0.50-1.39)$ \\
\hline
\end{tabular}


Table 5. Patterns of hypertension control based on recommended strategies

\begin{tabular}{|c|c|c|c|}
\hline \multirow[t]{2}{*}{ Hypertension } & \multicolumn{2}{|c|}{ Lifestyle strategy } & $\mathrm{p}$ \\
\hline & $\begin{array}{l}\text { Recommended } \\
\quad(95 \% \mathrm{CI})\end{array}$ & $\begin{array}{c}\text { non recommended } \\
(95 \% \mathrm{CI})\end{array}$ & \\
\hline Controlled & $37.3(35.4-39.1)$ & $39.1(34.2-44.2)$ & 0.510 \\
\hline Uncontrolled & $62.7(60.9-64.6)$ & $60.9(55.8-65.8)$ & \\
\hline
\end{tabular}

$(\mathrm{OR}=0.85, \% 95 \mathrm{CI}: 1.32-2.10)$ and smoking cessation $(\mathrm{OR}=0.51, \% 95 \mathrm{CI}: 0.81-0.31)$ was $85 \%$ and $15 \%$, respectively. In addition, the results of this study showed that in general, physician's recommendation odds on lifestyle strategies (weight loss, physical activity, healthier diet, and smoking cessation) to control hypertension was lower in patients living in the southeast compared to those who lived in other parts of the country (Table 4).

The results showed that obese people were more likely to receive physician's recommendation on body weight control $(\mathrm{OR}=2.26, \% 95 \mathrm{CI}: 1.92-2.67)$, physical activity $(\mathrm{OR}=1.30, \% 95 \mathrm{CI}: 1.12-1.54)$, and healthier diet $(\mathrm{OR}=$ 1.24, \%95 CI: 1.03-1.49) to control hypertension compare to patients with normal weight. However, patients with normal weight were received more recommendation on smoking cessation compare to obese people $(\mathrm{OR}=0.55$, \%95 CI: 0.39-0.76). In addition, diabetic participants were more recommended for body weight control $(\mathrm{OR}=1.55$, \%95 CI: 1.34-1.80), physical activity $(\mathrm{OR}=1.56, \% 95 \mathrm{CI}$ : 1.34-1.81), and healthy diet $(\mathrm{OR}=1.75, \% 95 \mathrm{CI}: 1.46$ 2.10) compared to the non-diabetic (Table 4).

The results of data analysis demonstrated that the association between lifestyle strategies recommendation and hypertension control was not statistically significant $(p=0.510)$ (Table 5). The finding showed that $60.9 \%$ of hypertensive patients who were recommended to change their lifestyle did not achieve BP control, indicating that the recommended lifestyle changes did not add a beneficial effect to BP control.

\section{Discussion}

This study was conducted to evaluate lifestyle strategies to control hypertension in adult patients and to determine the rate of physicians' recommendation for these strategies. The results showed that about three quarters of people with high blood pressure had been recommended to change their lifestyle for blood pressure control. Also, $41 \%$ of the participants reported that their physician had recommended only lifestyle modification for their BP, while $35 \%$ reported that their physician had recommended lifestyle changes and medications as part of their treatment. Evidence from previous studies suggests that these recommendations have a significant effect on lowering blood pressure. Smoking cessation and healthier diet were found to be the least and most frequent self-reported strategy, respectively, among hypertensive individuals who reported having been told by physicians to alter their lifestyle.

Also, results illustrated that rate of recommendation on weight loss, increased physical activity, and healthier diet were more among individuals with higher education, older age, women, obese individuals, diabetic persons, and rural dwellers compared to other people. Smoking cessation was recommended more to men, single people, urban residents, and the middle-aged. It was also found that $36.6 \%$ of individuals with hypertension were recommended to lose weight, and this recommendation was associated with diabetes, sex, marital status, and BMI. Women, diabetic persons, and married and obese individuals were recommended to change their lifestyle more than other people in the society. This finding is similar to that of previous studies $(9,16-18)$ and indicated that weight reduction reduces blood pressure (BP). Any 1\% decrease in body weight causes $1 \mathrm{mmHg}$ reduction in systolic blood pressure on average $(11,19)$.

Dietary habits play an important role in hypertension prevalence and control (20). Results showed that the most frequent recommended lifestyle modification was diet change. The results of this study are in line with those of George J. Fodor (6). Among hypertensive patients, 72\% said that their physician had recommended them to have a healthier diet. The reported diet recommendation among hypertensive patients varied from $45.4 \%$ in single patients to $79.7 \%$ in illiterate patients according to their characteristics. The chance of a physician recommending a healthier diet for hypertension control was higher in older individuals, women, and married participants, those residing in rural areas, illiterates, and obese people. One possible explanation for these findings is that older patients, those living in rural areas, illiterates, and obese people are especially sensitive to the effect of healthier diet on blood pressure $(9,18)$.

After healthier diet, recommending physical activity was the most frequent lifestyle modification. These findings are in agreement with those of previous studies (6). Increasing physical activity with a proper diet can lead to weight loss and, consequently, a decrease in blood pressure $(19,21,22)$. In this study, it was found that many factors were associated with physician recommendation to change life style in logistic regression. These factors were major risk factors for hypertension treatment and control (23-25). The chance of recommending physical activity by a physician was more among women, married, obese, and diabetic participants and those who lived in rural areas. Other cross sectional studies found similar results (25). Smokers have higher blood pressure levels and smoking causes an immediate increase in blood pressure (9). In this study, among $23.8 \%$ hypertensive patients, were told to stop smoking to control their hypertension. The men and patients who lived in urban areas were more likely to receive recommendation to stop smoking than women and patients who lived in urban areas. The results are consistent with previous studies that indicated chance of smoking was disproportionately different based on age, 
sex, and area of residency among people $(11,16-18)$.

Contrary to other studies $(6,11)$, the results of this study showed that lifestyle changes did not have a significant effect on BP control. One possible explanation for this discrepancy may be that in this study the rate of lifestyle strategies recommended by physicians was calculated, however, it was not determined whether people who had been recommended to change their lifestyle had actually adhered to the recommendations.

Another possibility may be that individuals with recommended lifestyle modification are less compliant in taking the prescribed medication. Finally, lifestyle modification as a recommended strategy for hypertension control might not have reached the standards set by efficacy guidelines, and thus had only minimal impact on BP.

For adequate power and unbiased estimates, data of this population-based study were obtained from STEPs program that included a national survey on a sample selected with a stratified multistage design. Furthermore, to our knowledge, this was the first report of lifestyle strategies recommended by physicians among Iranian hypertensive individuals.

The main limitation of this study was that the data were based on self-reported information. However, irrespective of the accuracy of a self-report, the findings provided valuable insight into the issue of physicians' recommendation on lifestyle strategies for BP control. Nevertheless, adherence to nonpharmacological strategies was not captured in STEPs to evaluate the impact of these strategies.

\section{Conclusion}

Because of the high prevalence of hypertension and its consequences, planning appropriate strategies to treat and control hypertension is essential. The results indicated that lifestyle changes have a main role in controlling high blood pressure. Because of the changes and transitions in lifestyle and dietary habits in the modern world and increased consumption of fast food and smoking, along with a reduction in physical activity due to technological development, policies and training programs should be developed to promote lifestyle modifications and increase physical activity. Furthermore, it seems that increasing people's knowledge about relevant strategies and encouraging them to adhere to these strategies can be effective in improving public health.

\section{Acknowledgments}

The authors thank those authors whose publications have been cited in this work and also thank NonCommunicable Diseases Research Center of Tehran University.

\section{Conflict of Interests}

The authors declare that they have no competing interests.

\section{References}

1. Members WG, Benjamin EJ, Blaha MJ, Chiuve SE, Cushman M, Das SR, et al. Heart disease and stroke statistics - 2017 update: a report from the American Heart Association. Circulation. 2017;135(10): e146.

2. Sata Y, Hering D, Head GA, Walton AS, Peter K, Marusic P, et al. Ambulatory arterial stiffness index as a predictor of blood pressure response to renal denervation. J Hypertens. 2018;36(6):1414-22.

3. Babaee Sis M, Ranjbaran S, Mahmoudi H, Baba Zadeh T, Moradi F, Mirzaeean K. The effect of educational intervention on lifestyle modification on blood pressure control in patients with hypertension. Journal of Education and Community Health. 2016;1(3):12-9.

4. Sim JJ, Shi J, Kovesdy CP, Kalantar-Zadeh K, Jacobsen SJ. Impact of achieved blood pressures on mortality risk and end-stage renal disease among a large, diverse hypertension population. J Am Coll Cardiol. 2014;64(6):588-97.

5. Brook RD, Appel LJ, Rubenfire M, Ogedegbe G, Bisognano JD, Elliott WJ, et al. Beyond medications and diet: alternative approaches to lowering blood pressure: a scientific statement from the American Heart Association. Hypertension. 2013: HYP. 0b013e318293645f.

6. Fodor GJ, McInnis NH, Helis E, Turton P, Leenen FH. Lifestyle Changes and Blood Pressure Control: A Community Based CrossSectional Survey (2006 Ontario Survey on the Prevalence and Control of Hypertension). J Clin Hypertens. 2009; 11(1):31-5.

7. Eckel RH, Jakicic JM, Ard JD, De Jesus JM, Miller NH, Hubbard VS, et al. 2013 AHA/ACC guideline on lifestyle management to reduce cardiovascular risk: a report of the American College of Cardiology/American Heart Association Task Force on Practice Guidelines. J Am Coll Cardiol. 2014;63(25 Part B):2960-84.

8. Semlitsch T, Jeitler K, Berghold A, Horvath K, Posch N, Poggenburg $\mathrm{S}$, et al. Long-term effects of weight-reducing diets in people with hypertension. The Cochrane Library. 2016.

9. Huang N, Duggan K. Lifestyle management of hypertension. Aust Prescr. 2008;31(6):150-3.

10. Stalsberg R, Pedersen AV. Effects of socioeconomic status on the physical activity in adolescents: a systematic review of the evidence. Scand J Med Sci Sports. 2010;20(3):368-83.

11. Oza R, Garcellano M. Nonpharmacologic management of

hypertension: what works? Am Fam Physician. 2015;91(11).

12. Esteghamati A, Abbasi M, Alikhani S, Gouya MM, Delavari A, Shishehbor $\mathrm{MH}$, et al. Prevalence, awareness, treatment, and risk factors associated with hypertension in the Iranian population: the national survey of risk factors for noncommunicable diseases of Iran. Am J Hypertens. 2008;21(6):620-6.

13. Djalalinia S, Modirian M, Sheidaei A, Yoosefi M, Zokaiee H, Damirchilu B, et al. Protocol Design for Large--Scale Cross-Sectional Studies of Surveillance of Risk Factors of Non-Communicable Diseases in Iran: STEPs 2016. Arch Iran Med. 2017;20(9).

14. American Diabetes Association, 2017. 2. Classification and diagnosis of diabetes. Diabetes care, 40(Supplement 1), pp.S11-S24.

15. Farzadfar F, Danaei G, Namdaritabar H, Rajaratnam JK, Marcus JR, Khosravi A, et al. National and subnational mortality effects of metabolic risk factors and smoking in Iran: a comparative risk assessment. Popul Health Metr. 2011;9(1):55.

16. Ho AK, Bartels CM, Thorpe CT, Pandhi N, Smith MA, Johnson HM. Achieving weight loss and hypertension control among obese adults: a US multidisciplinary group practice observational study. Am J Hypertens. 2016;29(8):984-91.

17. Sabaka P, Dukat A, Gajdosik J, Bendzala M, Caprnda M, Simko F. The effects of body weight loss and gain on arterial hypertension control: an observational prospective study. Eur J Med Res. 2017;22(1):43.

18. Zhao Y, Liu Y, Sun H, Sun X, Yin Z, Li H, et al. Association of long-term dynamic change in body weight and incident hypertension: The Rural Chinese Cohort Study. Nutrition. 2018; 54:76-82.

19. Duggan K, Anderson C, Arnolda L, Boyden A, Cowley D, Dart A, et al. Guide to the management of hypertension 2008-Assessing and managing raised blood pressure in adults. Guide Manag Hypertens. 2008;2008:1-34.

20. Kanauchi M, Kanauchi K. Diet quality and adherence to a healthy diet in Japanese male workers with untreated hypertension. BMJ Open. 2015;5(7): e008404.

21. Hegde SM, Solomon SD. Influence of physical activity on hypertension and cardiac structure and function. Curr Hypertens Rep. 2015;17(10):77.

22. Turi BC, Codogno JS, Fernandes RA, Monteiro HL. Physical activity, adiposity and hypertension among patients of public healthcare system. Rev Bra Epidemiol. 2014;17:925-37. 
23. Egan BM, Li J, Qanungo S, Wolfman TE. Blood pressure and cholesterol control in hypertensive hypercholesterolemic patients: NHANES 1988-2010. Circulation. 2013;128(1):29.

24. Egan BM, Zhao Y, Axon RN. US trends in prevalence, awareness, treatment, and control of hypertension, 1988-2008. JAMA. 2010;303(20):2043-50.

25. Liu X, Byrd JB, Rodriguez CJ. Use of physician-recommended nonpharmacological strategies for hypertension control among hypertensive patients. J Clin Hypertens. 2018;20(3):518-27. 